\title{
Mortality, Morbidity and Related Outcomes Following Perioperative Blood Transfusion in Patients with Major Orthopaedic Surgery: A Systematic Review
}

\author{
Susanne Müller Doris Oberle Ursula Drechsel-Bäuerle Jutta Pavel \\ Brigitte Keller-Stanislawski Markus B. Funk \\ Division Safety of Medicinal Products and Medical Devices, Paul-Ehrlich-Institut, Langen, Germany
}

\begin{abstract}
Keywords
Erythrocyte transfusion - Haemovigilance .

Immunomodulation - Non-haemolytic transfusion reactions $\cdot$ Peri-operative period $\cdot$ Restrictive and liberal transfusion regimes - Systematic review · Meta-analyses
\end{abstract}

\section{Summary}

Background: Benefits and risks of liberal and restrictive transfusion regimens are under on-going controversial discussion. This systematic review aimed at assessing both regimens in terms of pre-defined outcomes with special focus on patients undergoing major orthopaedic surgery. Methods: We performed a literature search for mortality, morbidity and related outcomes following peri-operative blood transfusion in patients with major orthopaedic surgery in electronic databases. Combined outcome measure estimates were calculated within the scope of meta-analyses including randomised clinical trials comparing restrictive versus liberal blood transfusion regimens (e.g. $\mathrm{MH}$ risk ratio, Peto odds ratio). Results: $\mathrm{A}$ total of 880 publications were identified 15 of which were finally included (8 randomised clinical trials (RCTs) with 3,693 patients and 6 observational studies with 4,244,112 patients). Regarding RCTs, no significant differences were detected between the transfusion regimes for all primary outcomes (30-day mortality, thromboembolic events, stroke/transitory ischaemic attack, myocardial infarction, wound infection and pneumonia) and a secondary outcome (length of hospital stay), whereas there was a significantly reduced risk of receiving at least one red

Susanne Müller and Doris Oberle contributed equally to this article.
(๑) 2018 S. Karger GmbH, Freiburg blood concentrate under a restrictive regimen. Conclusion: The results of this systematic review do not suggest an increased risk associated with either a restrictive or a liberal transfusion regimen in patients undergoing major orthopaedic surgery.

(c) 2018 S. Karger GmbH, Freiburg

\section{Introduction}

During the past 10 years, there was growing concern regarding potential risks associated with blood transfusions apart from the transmission of infectious agents. Benefits and risks of red blood cell (RBC) concentrate transfusion have intensively and controversially been discussed in various publications [1-4]. The occurrence of well-known serious adverse reactions (transfusion-related infection, haemolysis, allergic reaction, transfusion-related acute lung injury (TRALI) etc.) as well as the potential risk of suspected adverse reactions (immunological reaction, oncologic diseases, organ failure etc.) has been emphasised by several authors [2, 5-8].

Discussions are ongoing with respect to appropriate blood transfusion strategies balancing benefits and risks and defining adequate indications and appropriate thresholds for the transfusion of allogenic RBCs [5, 9].

In 2014, the German Medical Association (GMA) published an updated version of their cross-sectional guidelines applicable for therapy with blood components and plasma derivatives [10]. According to this GMA guideline, only haemoglobin $(\mathrm{Hb})$ blood levels $\leq 6 \mathrm{~g} / \mathrm{dl}$ are an absolute indication for transfusion of RBCs. Above this level, the indication for RBC transfusion depends on pre-existing risk factors (e.g. coronary artery disease), and a clear 
recommendation to avoid transfusion in patients without any further risk factors is given. For Hb levels ranging $\geq 8$ to $10 \mathrm{~g} / \mathrm{dl}$, RBC transfusion is only indicated in patients with symptoms of anaemic hypoxia (e.g. tachycardia, hypotension, transient ischaemic ECG changes etc.) [10].

Patients undergoing procedures in orthopaedic surgery for total hip arthroplasty (THA), total knee arthroplasty (TKA) or hip fracture surgery are the major recipients of allogenic blood transfusion in elective non-cancer surgery. Orthopaedic surgery procedures account for up to $10 \%$ of the RBCs transfused during surgical procedures [11]. The need for an increased transfusion rate grounds in the target organ 'bone' which tends to bleed heavily and bleeding is sometimes difficult to control due to its diffuse nature (in contrast to distinct bleedings from blood vessels). Furthermore, the typical population undergoing these procedures is mainly elderly with significant cardiovascular co-morbidity and of female gender which is associated with lower $\mathrm{Hb}$ levels and less blood volume.

In the two past decades, clinicians primarily followed two blood transfusion strategies: a more restrictive regimen setting the low $\mathrm{Hb}$ threshold value at $\leq 8 \mathrm{~g} / \mathrm{dl}$ and a more liberal one establishing the $\mathrm{Hb}$ threshold value at $\leq 10 \mathrm{~g} / \mathrm{dl}$. In addition, several blood-sparing techniques have been implemented, e.g. pre-donation of autologous blood, intra-operative blood cell saving and post-operative wound blood salvage for the purpose of reducing the need for allogenic blood transfusions.

Advocates of the more liberal regimen emphasise the benefits of $\mathrm{RBC}$ transfusions such as increase of oxygen supply, greater circulatory stability and a shorter rehabilitation period, whereas supporters of a restrictive strategy focus on potential risks such as increase of post-operative infection rate, increase of cardiovascular events (in particular, increase of thromboembolic events (TEE)), increase in length of stay (LOS) and last but not least a substantial increase in mortality [12-15].

In view of the above, it is deemed important to identify parameters of a safe use of both restrictive and liberal transfusion regimens in patients with orthopaedic surgery.

This systematic review aims at assessing the risk of peri-operative blood transfusion in patients undergoing major orthopaedic surgery. In our meta-analyses we focussed on a comparison between restrictive and liberal RBC transfusion strategies.

\section{Material and Methods}

Protocol and Registration

The study methodology was pre-specified and a protocol registered on PROSPERO (www.crd.york.ac.uk).

\section{Information Sources}

Records were identified by searching electronic databases. Articles from scanning reference lists of papers and reviews were also considered. The search was applied in Medline, Cochrane Central Register of Controlled Trials, Cochrane Database of Systematic Reviews, DAHTA Database, Database of Abstracts of Reviews of Effects, gms, gms Meetings, Health Technology Assessment Database, EMBASE, EMBASE Alert, ETHMED, CAB Abstracts, GLOBAL Health, BIOSIS Previews, BIOSIS Previews, ISTPB + ISTP/ISSHP and SciSearch.

\section{Identification}

The search was designed to be as sensitive as possible. No limits were applied for language.

The following search was used to identify potentially eligible reports:

Search ((((orthopaedic) AND blood) AND transfusion $))$ AND $((((()(((($ length of hospital stay) OR ((number) AND transfusions)) OR ((morbidity) AND postoperative)) OR infection) OR sepsis) OR ((postprocedure) AND sepsis)) OR ((thromboembolic) AND events)) OR ((thrombosis) AND postoperative)) OR ((embolism) AND postoperative)) OR death) OR fatal) OR mortality)

Search Details:

((('orthopaedic'[All Fields] OR 'orthopedics'[MeSH Terms] OR 'orthopedics'[All Fields] OR 'orthopedic'[All Fields]) AND ('blood'[Subheading] OR 'blood'[All Fields] OR 'blood'[MeSH Terms])) AND ('blood transfusion'[MeSH Terms] OR ('blood'[All Fields] AND 'transfusion'[All Fields]) OR 'blood transfusion'[All Fields] OR 'transfusion'[All Fields])) AND (((()((()(('length of stay'[MeSH Terms] OR ('length'[All Fields] AND ‘stay'[All Fields]) OR 'length of stay'[All Fields] OR ('length'[All Fields] AND 'hospital'[All Fields] AND 'stay'[All Fields]) OR 'length of hospital stay'[All Fields]) OR (number[All Fields] AND ('blood transfusion'[MeSH Terms] OR ('blood'[All Fields] AND 'transfusion'[All Fields]) OR 'blood transfusion'[All Fields] OR 'transfusions'[All Fields]))) OR (('epidemiology'[Subheading] OR 'epidemiology'[All Fields] OR 'morbidity'[All Fields] OR 'morbidity'[MeSH Terms]) AND ('postoperative period'[MeSH Terms] OR ('postoperative'[All Fields] AND 'period'[All Fields]) OR 'postoperative period'[All Fields] OR 'postoperative'[All Fields]))) OR ('infection'[MeSH Terms] OR 'infection'[All Fields])) OR ('sepsis'[MeSH Terms] OR 'sepsis'[All Fields])) OR (postprocedure[All Fields] AND ('sepsis'[MeSH Terms] OR 'sepsis'[All Fields]))) OR (thromboembolic[All Fields] AND events[All Fields])) OR (('thrombosis'[MeSH Terms] OR 'thrombosis'[All Fields]) AND ('postoperative period'[MeSH Terms] OR ('postoperative'[All Fields] AND 'period'[All Fields]) OR 'postoperative period'[All Fields] OR 'postoperative'[All Fields]))) OR (('embolism'[MeSH Terms] OR 'embolism'[All Fields]) AND ('postoperative period'[MeSH Terms] OR ('postoperative'[All Fields] AND 'period'[All Fields]) OR 'postoperative period'[All Fields] OR 'postoperative'[All Fields]))) OR ('death'[MeSH Terms] OR 'death'[All Fields])) OR fatal[All Fields]) OR ('mortality'[Subheading] OR 'mortality'[All Fields] OR 'mortality'[MeSH Terms]))

Additional records were identified by checking reference lists. Duplicates were removed.

\section{Screening}

Title and abstract screening were conducted independently by two individuals on all of the articles retrieved during the search. Only references that both reviewers agreed to be not relevant to the review were excluded.

Screening questions were:

- Is this a primary study?

- Was blood product administered peri-operatively in patients undergoing orthopaedic surgery?

- Did the study examine the risk of blood transfusion?

- Was the publication date within the period 1995-2015? Full texts for the remaining references were obtained.

\section{Eligibility Check}

The obtained full texts were independently checked for eligibility by two individuals according to the criteria specified below:

- Design: Randomised controlled trial (RCT) / prospective non-randomised interventional study / cohort/ case-control / cross-sectional / case series / signal detection report / case report.

- Participants: Patients undergoing major orthopaedic surgery.

- Study population source: Hospital patients / health-related database.

- Interventions: Transfusion of allogenic RBCs / autologous blood / whole blood; restrictive / liberal transfusion strategy.

- Blood component specifications: Buffy coat-depleted / leucocyte-depleted / undepleted / washed / irradiated / not otherwise specified (NOS). 

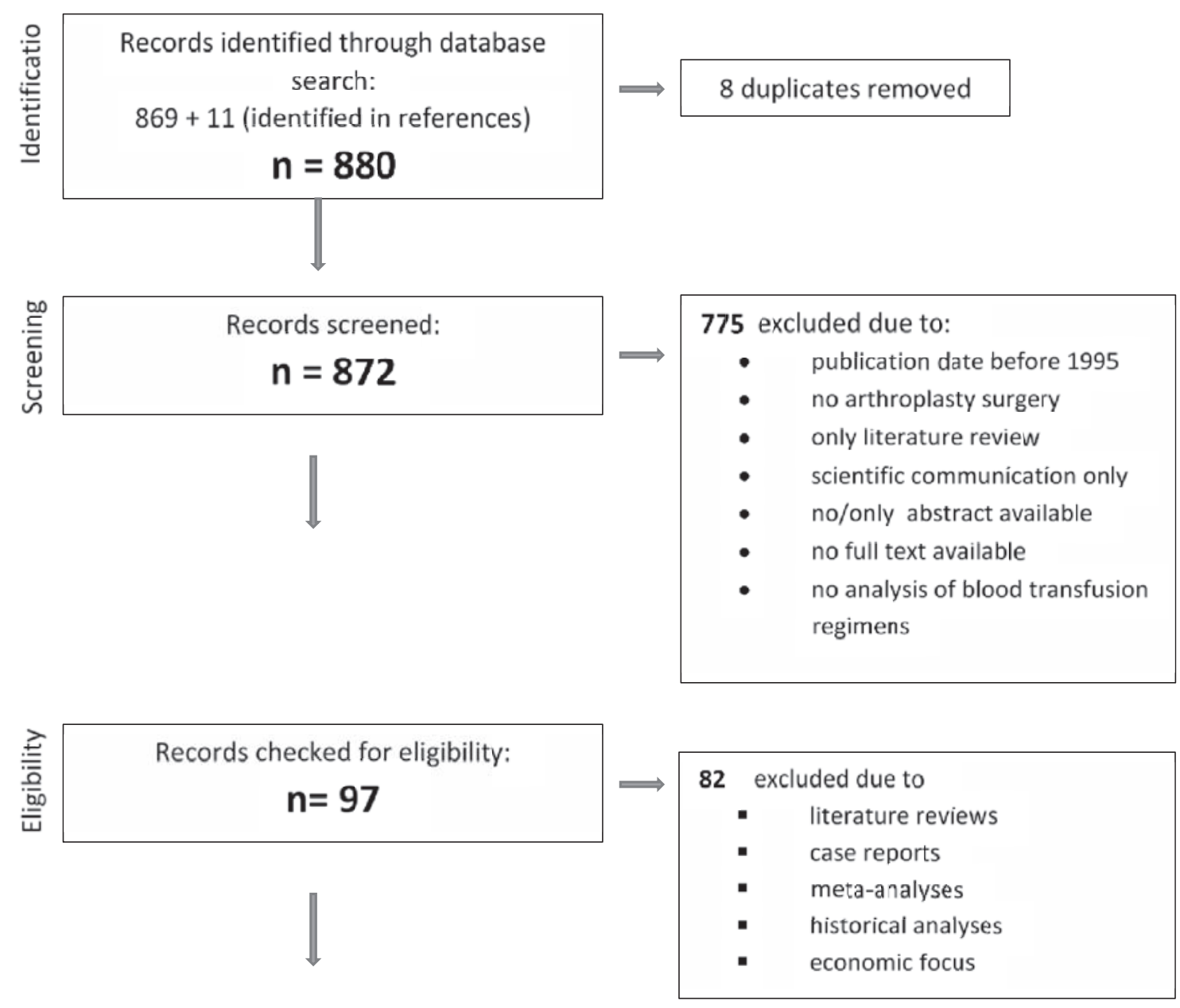

Fig. 1. Flow chart. ${ }^{*} 1$ post-hoc analysis of a randomised clinical trial included based on the same data.

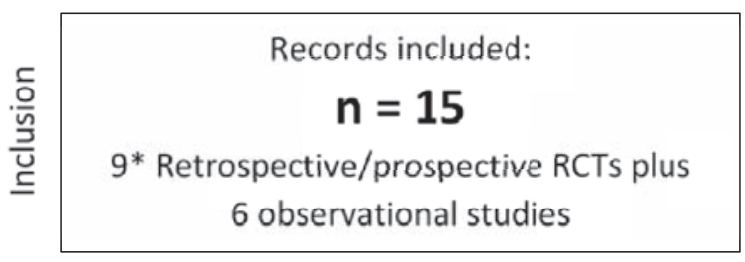

- Outcomes: Post-operative mortality, post-operative morbidity, post-operative infection, TEE, LOS and re-admission.

Records that did not fulfil the eligibility criteria were excluded.

\section{Data Extraction}

Data extraction tables and forms were developed to standardise the information extracted from each of the eligible studies. Data from included records were extracted and then independently cross-checked by another individual.

\section{Data Items}

The following data from each included record were extracted: general characteristics (study design, study location(s), publication type, source of funding, possible conflict of interest, linked or duplicate publications identified by search, references identified in text that should be additionally screened), study participants (total, study population size, study population source, eligibility criteria, number of eligible subject), recruitment method, year recruitment started, year recruitment ended, number recruited, number in study arms, reason for non-response, pre-operative anaemia, pre-operative co-morbidity), study intervention (intervention type, co-intervention, period of blood transfusion, blood component specifications, timing of blood transfusion, volume of transfused blood, type of blood management, transfusion triggers), intra-opera- tive events (duration of surgery, intra-operative complications, intra-operative blood transfusion) and outcome (outcome type, study definition of outcome, number and type of participants evaluated for this outcome, method of ascertainment of outcome, total duration of follow-up to determine this outcome, outcome measurement, occurrence of zero events).

\section{Risk of Bias in Individual Studies}

Risk of bias has been assessed for RCTs during the data extraction process by two reviewers separately, focussing on the following risks: confounding, selection, information, and reporting biases, as well as bias due to departure from intervention and missing data. The reviewers were guided by signalling questions and made a judgement on the level of each type of bias found within the RCT. We completed a 'Risk of bias' table for each RCT. Regarding observational studies no formal bias assessment was performed due to the strictly observational approach which implies considerable bias risks per se that can be ascribed to a lack of control for potential influencing factors according to a predefined study protocol.

\section{Statistical Analysis}

With respect to the outcomes RBC transfusion, 30-day mortality, TEE, stroke/ transient ischemic attack (TIA), cardiovascular event/death, myocardial 


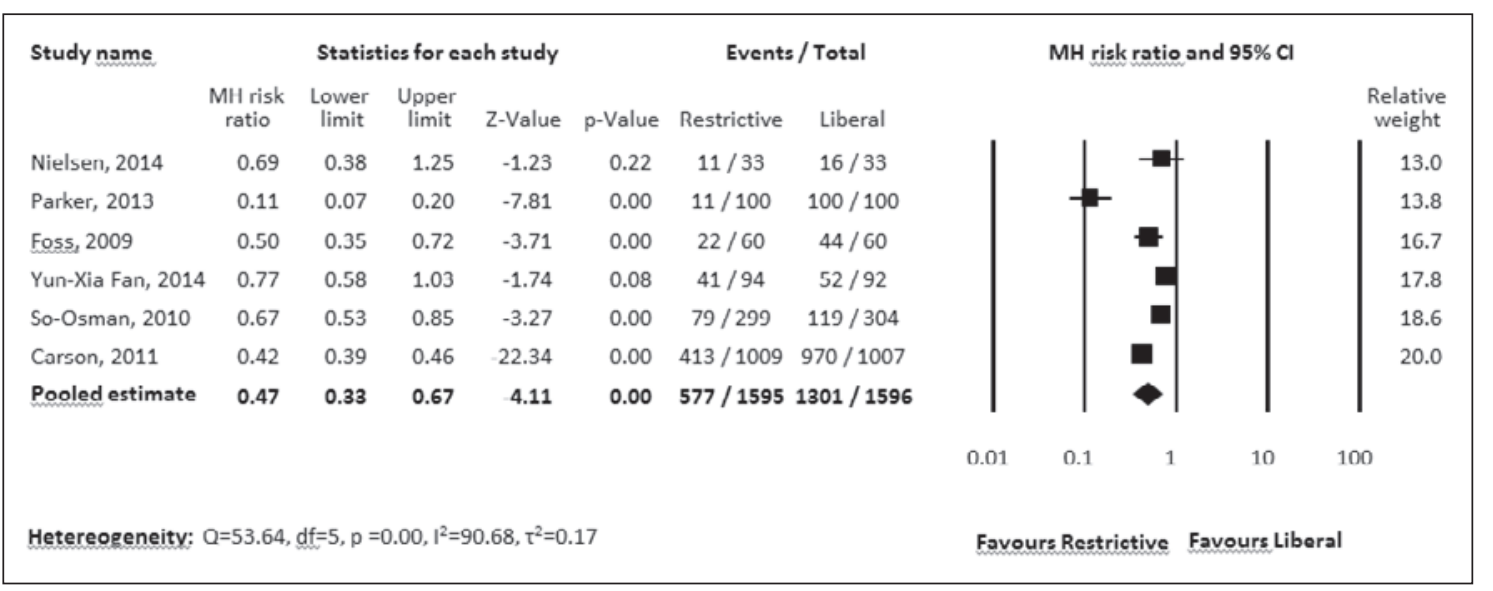

infarction, wound infection and pneumonia, the Maentel-Haenzel risk-ratio (MH (RR)) with corresponding 95\% confidence intervals (CI) was calculated for each RCT comparing a restrictive versus a liberal blood transfusion regimen. When the event rate was low, we considered using the Peto odds ratio as effect measure when criteria for this method were fulfilled. With respect to LOS, the difference of means and 95\% CI were computed for each RCT comparing a restrictive versus a liberal blood transfusion regimen.

\section{Sensitivity Analyses}

Meta-analyses were repeated with exclusion of one RCT [14] in order to assess the effect of selective inclusion of patients with pre-existing cardiovascular disease and/or cardiovascular risk factors.

\section{Data Synthesis}

Regarding qualitative outcomes, meta-analyses were performed calculating a pooled MH RR with corresponding 95\% CI for clinical studies comparing a restrictive versus a liberal blood transfusion regimen. We calculated pooled Peto odds ratios for outcomes with event rates less than $1 \%$. With respect to continuous outcomes, the pooled difference of means and 95\% CI were computed for RCTs comparing a restrictive versus a liberal blood transfusion regimen within the scope of a further meta-analysis. Except for outcomes with Peto odds ratio used as effect measure, data were analysed using a random-effects model because of the anticipated clinical heterogeneity of the studies. For each meta-analysis performed, a forest plot was provided. For observational studies, no data synthesis was performed due to the lack of comparable interventions and outcomes precluding the calculation of combined estimates.

\section{Heterogeneity}

Heterogeneity was assessed using the Q-test which informs about the presence versus the absence of heterogeneity and the $\mathrm{I}^{2}$ statistic quantifying the extent of heterogeneity [16]. In addition, as an estimate of the between-study variance in a random-effects meta-analysis, tau-squared $\left(\tau^{2}\right)$ was computed.

\section{Statistical Software}

The meta-analyses were performed using the software Comprehensive Meta-Analysis, version 2.2.064 (Biostat, Englewood, NJ, USA).

\section{Results}

The literature search retrieved 869 publications (fig. 1). Eleven publications were additionally found in references, so a total of 880 publications were identified. Eight duplicates were removed leaving 872 publications screened by 2 independent medical reviewers according to pre-specified eligibility criteria. 775 publications were excluded due to the unavailability of the full text, a publication date prior to 1995 , or a study population not having undergone arthroplasty/major orthopaedic surgery, or because studies did not analyse different blood transfusion regimens or were literature reviews or scientific communication referring to publications. The remaining 97 publications were checked for eligibility by abstract and full text review.

Following a second check for eligibility, further 82 records were excluded because they referred to meta-analyses or case reports, or focused on history or economic impacts of different transfusion regimens.

15 publications referring to 14 studies (8 RCTs, 6 observational studies) were finally included in the systematic review. Within the RCT group, two publications $[17,18]$ referred to the same RCT but highlighted different aspects warranting the inclusion of both.

The included 8 RCTs (table 1) performed between 2000 and 2013 (thus comparable RBC standards may be assumed, (e.g. leucocyte-reduced RBCs) differ regarding sample size and study population.

In the 8 RCTs, 1,848 participants were randomised to the restrictive transfusion regimen with $\mathrm{Hb}$ blood threshold $<8 \mathrm{~g} / \mathrm{dl}$ and 1,845 participants to the liberal transfusion regimen with $\mathrm{Hb}$ blood threshold $>8-10 \mathrm{~g} / \mathrm{dl}$. The mean age of participants was similar across all trials, ranging from 70 to 86 years. In the majority of the clinical trials gender distribution was skewed towards female participants (72.2\%), only 3 clinical trials showed a balanced gender ratio.

In the observational studies, the mean age ranged from 64 to 85 years and, like in the RCTs, females (64\%) clearly outweighed males.

For most of the RCTs, risk of bias was rated low for the main criteria: confounding, selection, departure from intervention, information, missing data and conflict of interest. Only a few moderate ratings occurred: for confounding [19, 20], selection [19], information $[15,20,21]$, missing data $[22,23]$, and departure from interventions [19-21]. Overall, the RCTs performed by Parker [19] and Fan et al. [20] received 3 moderate ratings each.

Six observational studies which showed considerable heterogeneity regarding protocol, study period, population size, interventions and outcomes were included (table 2). 


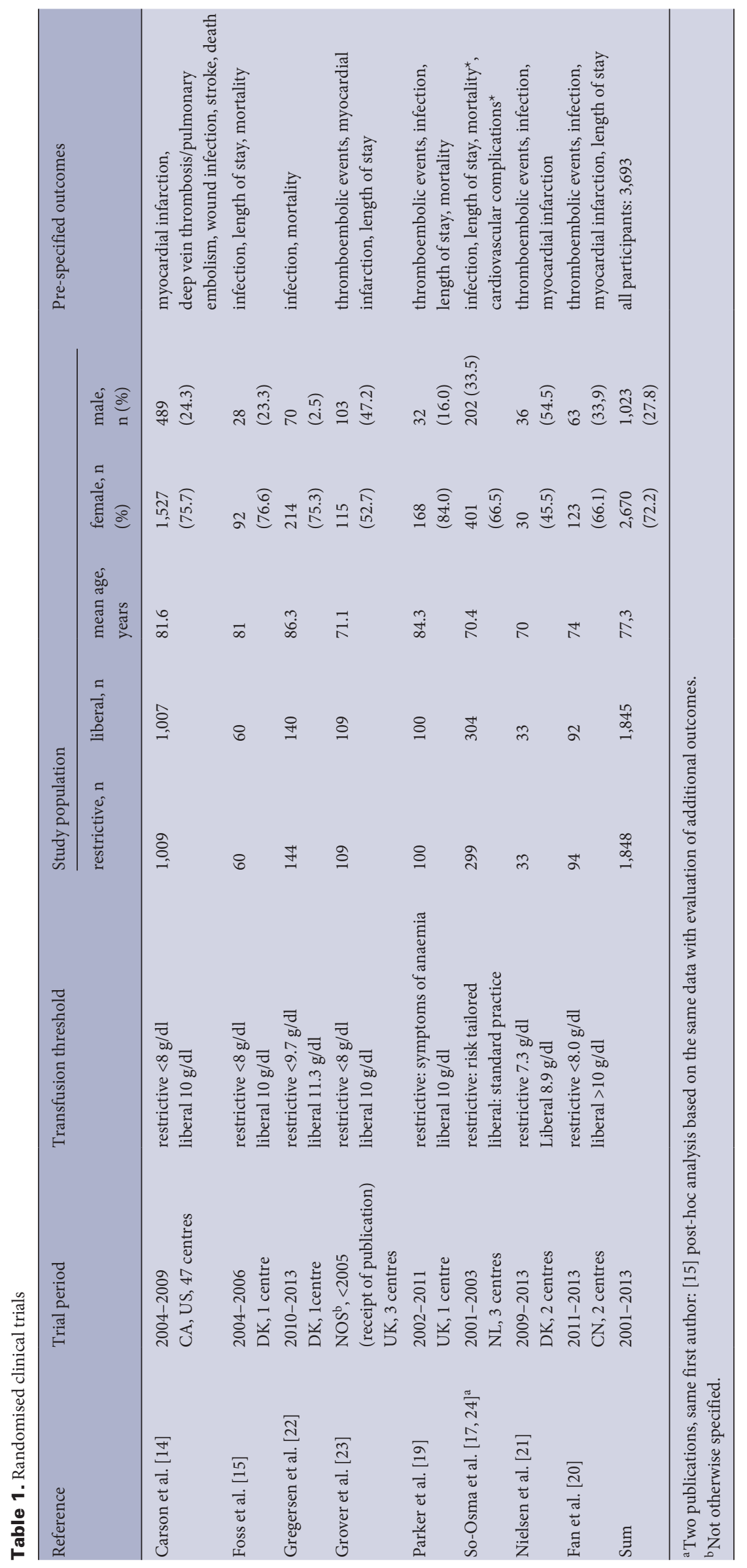


Fig. 3. Meta-analysis of clinical trials evaluating the risk of 30-day mortality comparing a restrictive and liberal blood transfusion regimen (random effects model) and forest plot.

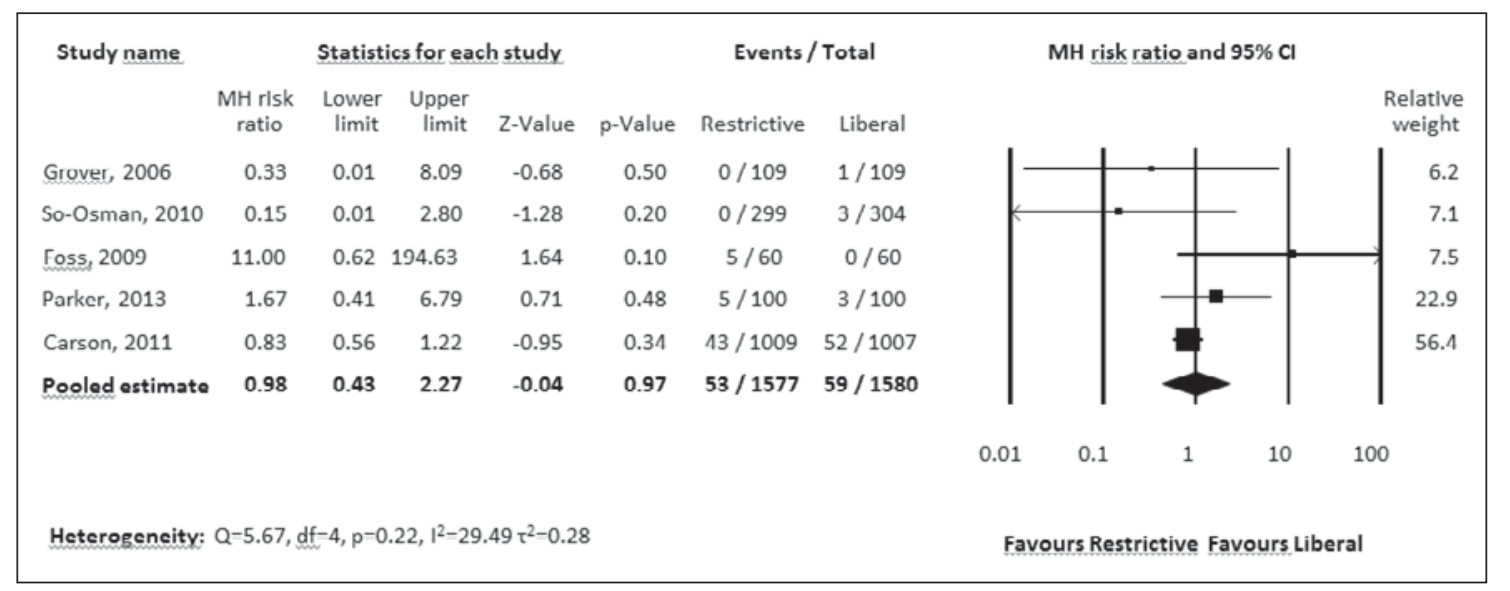

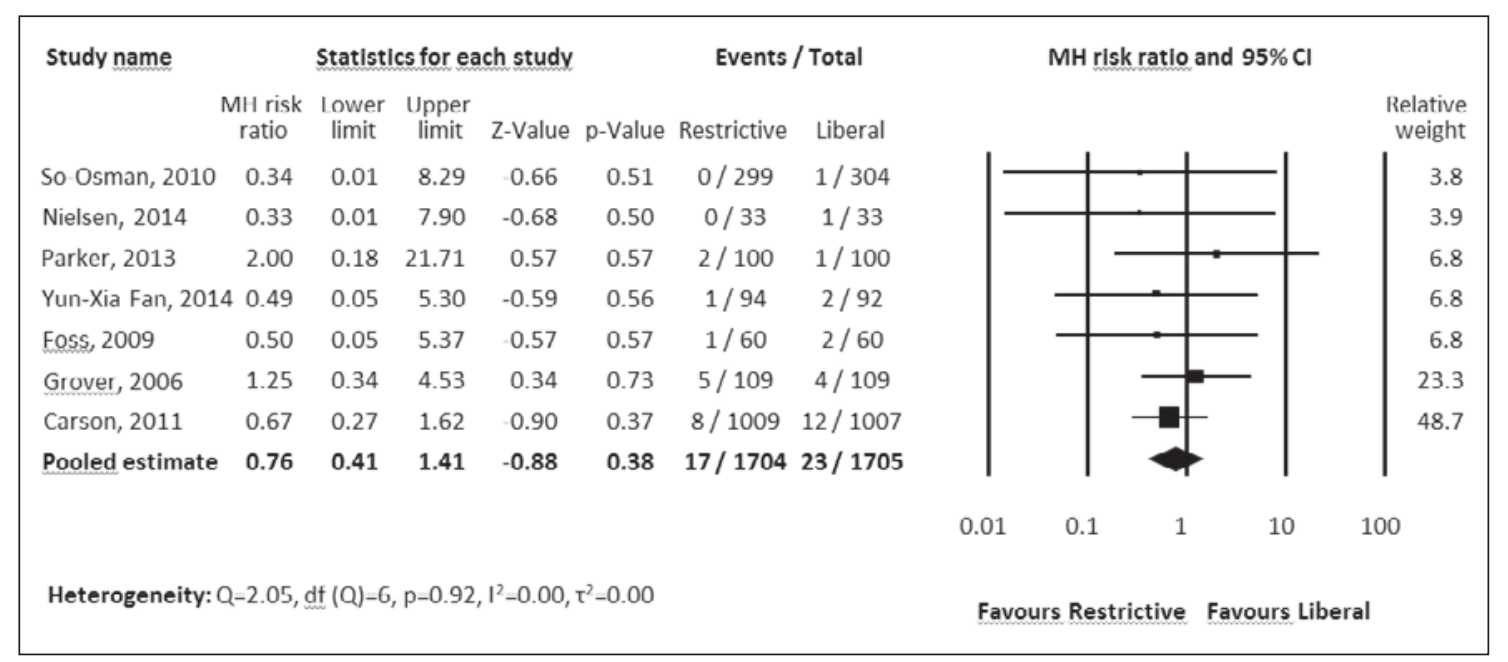

Fig. 4. Meta-analysis of clinical trials evaluating the risk of thromboembolic events comparing a restrictive and liberal blood transfusion regimen (random effects model) and forest plot.

\section{Outcome Analyses RCTs}

\section{Risk of Receiving a RBC Transfusion}

Regarding the risk of receiving a RBC transfusion, 6 RCTs were analysed including 3,191 participants - 1,595 patients in the restrictive and 1,596 in the liberal transfusion regimen group (fig. 2). Likelihood of receiving a transfusion was statistically different between the 2 transfusion regimens ( $\mathrm{RR} 0.47,95 \% \mathrm{CI} 0.33-0.67, \mathrm{Z}=-4.11, \mathrm{p}<0.01$ ) in favour of the restrictive group. For this group likelihood to receive a transfusion was about $50 \%$ decreased compared to the patients of the liberal group. Heterogeneity between the trials was also statistically significant $\left(\mathrm{Q}=53.64, \mathrm{df}=5, \mathrm{p}<0.01, \mathrm{I}^{2}=90.68, \tau^{2}=0.17\right)$.

\section{0-Day Mortality}

For the evaluation of 30-day mortality, data of 6 RCTs were included. However, in the study by Nielsen et al. [21], no fatalities occurred within the 30-day range so that the RR has been calculated based on 5 RCTs including 3,157 participants reporting events for this outcome in at least one study group (fig. 3). No significant difference was found between the restrictive and liberal transfusion strategies (RR $0.98,95 \%$ CI $0.43-2.27, \mathrm{Z}=-0.04, \mathrm{p}=$ 0.97). This result was supported by a sufficient homogeneity of study data $\left(\mathrm{Q}=5.67, \mathrm{df}=4, \mathrm{p}=0.22, \mathrm{I}^{2}=29.49 \tau^{2}=0.28\right)$.

\section{Risk of TEE}

Seven RCTs reported data for TEE in 3,409 participants (fig. 4). Though, in 5 out of the 7 RCTs, more TEE occurred in the liberal transfusion group, the absolute number of those events was low, with the difference between the groups being negligible. Consequently, no difference was found between the restrictive and liberal transfusion strategies (RR 0.76, 95\% CI 0.41-1.41, Z = $-0.88, p=0.38$ ) in terms of this outcome. Data are supported by a low degree of heterogeneity $\left(\mathrm{Q}=2.05, \mathrm{df}=6, \mathrm{p}=0.92, \mathrm{I}^{2}=0.00\right.$, $\left.\tau^{2}=0.00\right)$.

\section{Risk of Stroke / TIA}

Regarding stroke and TIA, data of 2,522 participants from 4 RCTs were analysed (fig. 5). The majority of events occurred in the trial of Carson et al. [14] that reported 5 stroke events for the liberal group, whereas only 1 event was reported for the restrictive group. Though the forest plot reflects a trend towards a higher rate in patients with a liberal transfusion regimen, no significant difference was confirmed (RR $0.36,95 \%$ CI 0.12 to $1.13, \mathrm{Z}=-1.75, \mathrm{p}=$ $0.08)$ on the basis of a low degree of heterogeneity $(\mathrm{Q}=0.99, \mathrm{df}=3$, $\left.\mathrm{p}=0.80, \mathrm{I}^{2}=0.00, \tau^{2}=0.00\right)$. 


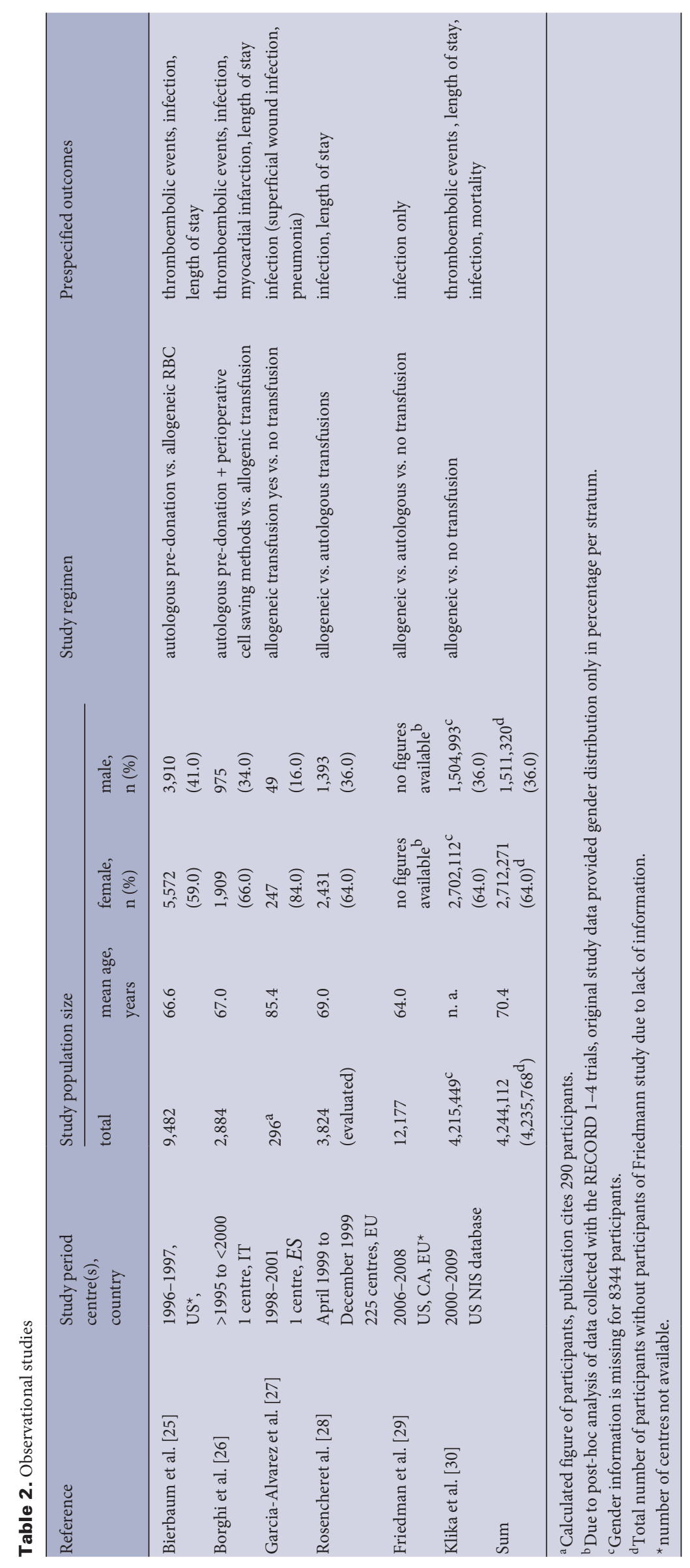


Fig. 5. Meta-analysis of clinical trials evaluating the risk of stroke/ transient ischemic attack comparing a restrictive and liberal blood transfusion regimen (fixed effects model) and forest plot.

Fig. 6. Meta-analysis of clinical trials evaluating the risk of myocardial infarction comparing a restrictive and liberal blood transfusion regimen (random effects model) and forest plot.

Fig. 7. Meta-analysis of clinical trials evaluating the risk of wound infection comparing a restrictive and liberal blood transfusion regimen (random effects model) and forest plot.

\begin{tabular}{|c|c|c|c|c|c|c|c|c|c|c|c|}
\hline \multirow[t]{2}{*}{ Study name } & \multirow[b]{2}{*}{$\begin{array}{c}\text { Peto } \\
\text { odds ratio }\end{array}$} & \multicolumn{3}{|c|}{ Statistics for each study } & \multirow[b]{2}{*}{$\mathrm{p}$-Value } & \multicolumn{2}{|c|}{ Events / Total } & \multicolumn{3}{|c|}{ Peto odds ratio and $95 \% \mathrm{Cl}$} & \multirow[b]{2}{*}{$\begin{array}{l}\text { Relative } \\
\text { weight }\end{array}$} \\
\hline & & $\begin{array}{l}\text { Lower } \\
\text { limit }\end{array}$ & $\begin{array}{c}\text { Upper } \\
\text { limit }\end{array}$ & z-Value & & Restrictive & Liberal & & & & \\
\hline Parker, 2013 & 0.14 & 0.00 & 6.82 & -1.00 & 0.32 & $0 / 100$ & $1 / 100$ & & & & 8.4 \\
\hline Foss, 2009 & 1.00 & 0.06 & 16.18 & 0.00 & 1.00 & $1 / 60$ & $1 / 60$ & & & 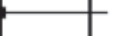 & 16.6 \\
\hline Yun-Xia Fan, 2014 & 0.50 & 0.05 & 4.85 & -0.60 & 0.55 & $1 / 94$ & $2 / 92$ & & & & 24.9 \\
\hline Carson, 2011 & 0.26 & 0.05 & 1.30 & -1.64 & 0.10 & $1 / 1009$ & $5 / 1007$ & & & & 50.1 \\
\hline \multirow[t]{2}{*}{ Pooled estimate } & 0.36 & 0.12 & 1.13 & -1.75 & 0.08 & $3 / 1263$ & $9 / 1259$ & & & & \\
\hline & & & & & & & & 0.01 & 0.1 & 10 & 100 \\
\hline \multicolumn{8}{|c|}{ Heterogeneity: $\mathrm{Q}=0.99, \mathrm{df}(\mathrm{Q})=3, \mathrm{p}=0.80, \mathrm{I}^{2}=0.00, \mathrm{\tau}^{2}=0.00$} & Fave & rs Restrictive & Favours Libe & \\
\hline
\end{tabular}

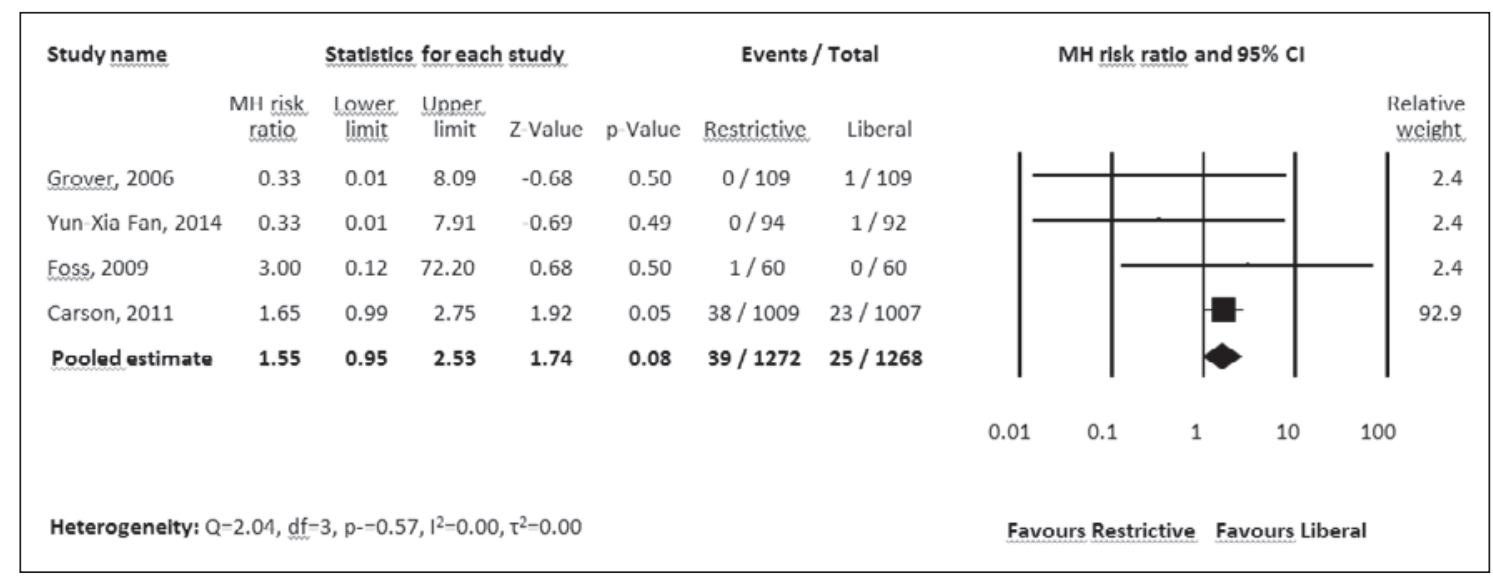

\begin{tabular}{|c|c|c|c|c|c|c|c|c|c|c|c|c|}
\hline \multirow[t]{2}{*}{ Study name } & \multicolumn{5}{|c|}{ Statistics for each study } & \multicolumn{2}{|c|}{ Events / Total } & \multicolumn{5}{|c|}{$\mathrm{MH}$ risk ratio and $95 \% \mathrm{Cl}$} \\
\hline & $\begin{array}{c}\text { MH risk } \\
\text { ratio }\end{array}$ & $\begin{array}{c}\text { Lower } \\
\text { limit }\end{array}$ & $\begin{array}{c}\text { Upper } \\
\text { limit }\end{array}$ & Z-Value & $\mathrm{p}$-Value & Restrictive & Liberal & & & & & $\begin{array}{r}\text { Relative } \\
\text { weight }\end{array}$ \\
\hline Nielsen, 2014 & 0.33 & 0.01 & 7.90 & -0.68 & 0.50 & $0 / 33$ & $1 / 33$ & & & & & 3.2 \\
\hline Foss, 2009 & 0.14 & 0.01 & 2.71 & -1.30 & 0.19 & $0 / 60$ & $3 / 60$ & & & & & 3.7 \\
\hline Grover, 2006 & 1.00 & 0.14 & 6.97 & 0.00 & 1.00 & $2 / 109$ & $2 / 109$ & & & & & 8.4 \\
\hline Yun-Xia Fan, 2014 & 0.65 & 0.11 & 3.82 & -0.47 & 0.64 & $2 / 94$ & $3 / 92$ & & & & & 10.2 \\
\hline So-Osman, 2010 & 0.61 & 0.22 & 1.66 & -0.97 & 0.33 & $6 / 299$ & $10 / 304$ & & & & & 31.9 \\
\hline Carson, 2011 & 0.57 & 0.24 & 1.35 & -1.27 & 0.20 & $8 / 1009$ & $14 / 1007$ & & & 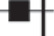 & & 42.6 \\
\hline \multirow[t]{2}{*}{ Pooled estimate } & 0.58 & 0.33 & 1.02 & -1.90 & 0.06 & $18 / 1604$ & $33 / 1605$ & & & & & \\
\hline & & & & & & & & 0.01 & 0.1 & 1 & 10 & 100 \\
\hline \multicolumn{8}{|c|}{ Heterogeneity: $\mathrm{Q}=1.33, \mathrm{df}=5, \mathrm{p}=0.93, \mathrm{l}^{2}=0.00, \tau^{2}=0.00$} & \multicolumn{5}{|c|}{ Favours Restrictive } \\
\hline
\end{tabular}

\section{Risk of Myocardial Infarction}

Regarding myocardial infarction, no significant difference between the restrictive and liberal transfusion strategies was demonstrated (RR 1.55, 95\% CI 0.95-2.53, $\mathrm{Z}=1.74, \mathrm{p}=0.08$ ). On the basis of 4 RCTs with 2,540 participants, 39 patients with a restrictive regimen and 25 patients with a liberal regimen developed a myocardial infarction within 30 days after transfusion (fig. 6). No distinct heterogeneity restricts the statistical analysis $(\mathrm{Q}=2.04$, df $=3, \mathrm{p}=0.57, \mathrm{I}^{2}=0.00, \tau^{2}=0.00$ ).

Of note, the statistical significance in favour of the liberal regimen was rather narrowly missed $(\mathrm{p}=0.08)$ like in the trial by Carson et al. [14] $(p=0.05)$. However, in the trial of Carson et al. [14] that contributes with the highest relative weight (relative weight: 92.9\%), included only participants indicative of a history of cardiovascular disease. To evaluate the actual impact of selection bias, a sensitivity analysis was performed with this trial removed (see below).

\section{Risk of Wound Infection}

Seven RCTs reported data for wound infection, but in Parker et al. 2013 [19] no events were reported, leaving 6 trials with 3,209 patients (fig. 7). There was no difference between the restrictive and liberal transfusion strategies (RR $0.58,95 \%$ CI $0.33-1.02, \mathrm{Z}=$ $-1.90, p=0.06)$ though a trend in favour of the restrictive regimen 
Fig. 8. Meta-analysis of clinical trials evaluating the risk of pneumonia comparing a restrictive and liberal blood transfusion regimen (random effects model) and forest plot.

Fig. 9. Meta-analysis of the difference in means of length of stay comparing a restrictive and liberal blood transfusion regimen (random effects model) and forest plot.

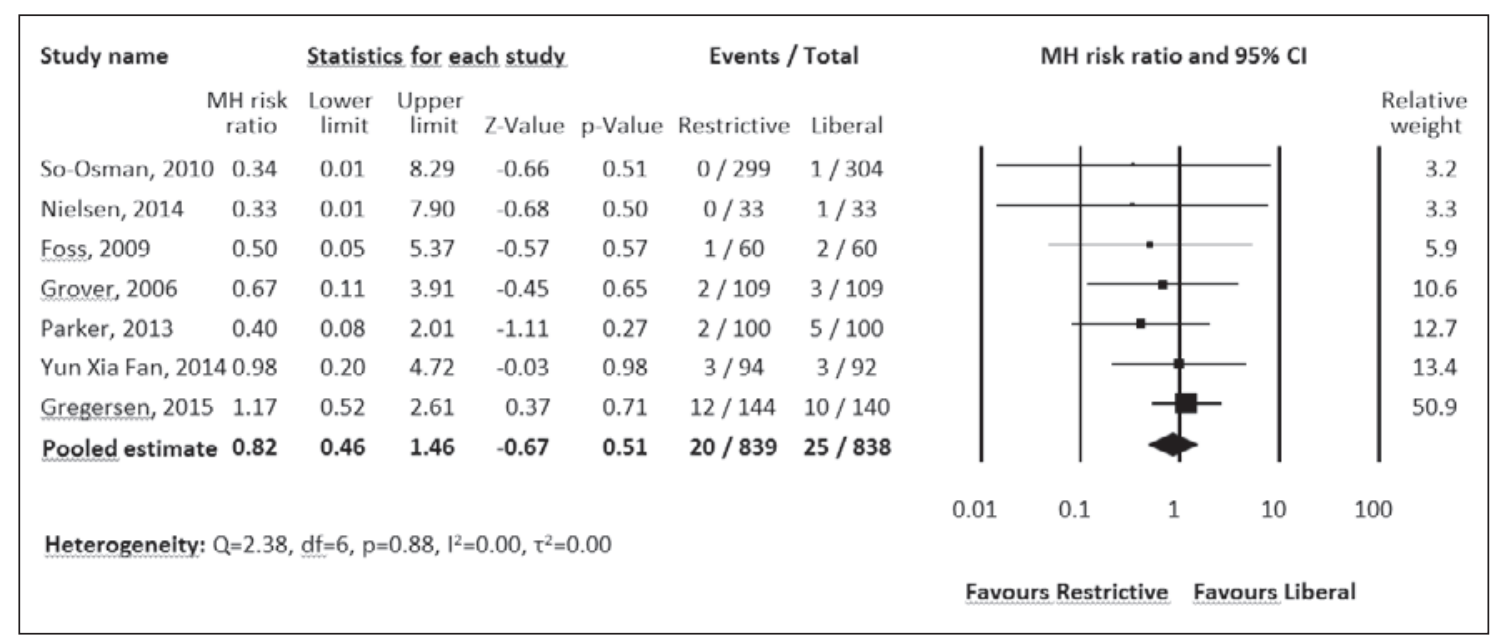

\begin{tabular}{|c|c|c|c|c|c|c|c|c|c|c|c|c|c|}
\hline \multirow[t]{2}{*}{ Study name } & & \multicolumn{5}{|c|}{ Statistics for each study } & & \multicolumn{2}{|c|}{ Sample size } & \\
\hline & \multicolumn{2}{|c|}{ Difference Standard Lower Upper } & Varianc & $\begin{array}{l}\text { Lower } \\
\text { celimit }\end{array}$ & Upper & z-Value & p-Value & Restricti & ve Liberal & \multicolumn{4}{|c|}{$\begin{aligned} \text { Difference in means and } 95 \% \mathrm{Cl} \\
\\
\begin{aligned} \text { Relative } \\
\text { weight }\end{aligned}\end{aligned}$} \\
\hline Parker, 2013 & 1.50 & 3.20 & 10.25 & -4.78 & 7.78 & 0.47 & 0.64 & 100 & 100 & & & & 0.6 \\
\hline Foss, 2009 & -1.40 & 2.50 & 6.23 & -6.29 & 3.49 & -0.56 & 0.57 & 60 & 60 & & & & 1.0 \\
\hline So-Osman, 2010 & 0.60 & 0.54 & 0.30 & -0.47 & 1.67 & 1.10 & 0.27 & 299 & 304 & & & 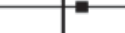 & 21.3 \\
\hline Yun-Xia Fan, 2014 & $14-0.50$ & 0.49 & 0.24 & -1.46 & 0.46 & -1.02 & 0.31 & 94 & 92 & & & & 26.1 \\
\hline Carson, 2011 & 0.03 & 0.35 & 0.12 & -0.66 & 0.72 & 0.08 & 0.94 & 1009 & 1007 & & & & 50.9 \\
\hline \multirow[t]{2}{*}{ Pooled estimate } & 0.01 & 0.25 & 0.06 & -0.49 & 0.50 & 0.02 & 0.98 & 1562 & 1563 & & & & \\
\hline & & & & & & & & & & -1.00 & -0.50 & 0.50 & 1.00 \\
\hline \multicolumn{14}{|c|}{ Heterogeneity: $Q=2.80$. df $=4 . p=0.59 . I^{2}=0.00 . \tau^{2}=0.00$} \\
\hline
\end{tabular}

was obvious. Heterogeneity between these trials was not statistically significant $\left(\mathrm{Q}=1.33, \mathrm{df}=5, \mathrm{p}=0.93, \mathrm{I}^{2}=0.00, \tau^{2}=0.00\right)$.

\section{Risk of Pneumonia}

Seven RCTs reported data for pneumonia in 1,677 participants (fig. 8). There was no difference between the restrictive and liberal transfusion strategies (RR 0.82, 95\% CI 0.46-1.46, $\mathrm{Z}=-0.67, \mathrm{p}=$ $0.51)$ in terms of this outcome. This result was supported by a sufficient homogeneity of study data $\left(\mathrm{Q}=2.38, \mathrm{df}=6, \mathrm{p}=0.88, \mathrm{I}^{2}=\right.$ $\left.0.00, \tau^{2}=0.00\right)$.

\section{LOS}

Regarding the parameter LOS, data of 5 RCTs with 3,125 participants were included in the meta-analysis (fig. 9). There was no difference between the restrictive and liberal transfusion strategies (difference in means $0.01,95 \% \mathrm{CI},-0.49$ to 0.50 ) in terms of this outcome. Heterogeneity between these trials was not statistically significant $\left(\mathrm{Q}=2.80, \mathrm{df}=4, \mathrm{p}=0.59,=0.00, \tau^{2}=0.00\right)$

\section{Sensitivity Analyses}

By excluding the trial of Carson et al. [14] which selectively investigated a subpopulation of orthopaedic surgery patients (subjects with pre-existing cardiac disease and/or with cardiovascular risk factors) and which was the study with the highest relative weight in the meta-analysis, the combined results did not change except for the outcome 'myocardial infarction' where the combined estimate was shifted in favour of the restricted transfusion regimen (combined RR $0.69,95 \%$ CI 0.11 to $4.34, \mathrm{Z}=-0.40, \mathrm{p}=0.69)$, with heterogeneity remaining negligible $\left(\mathrm{Q}=1.23, \mathrm{df}=2, \mathrm{p}=0.54, \mathrm{I}^{2}<0.00, \tau^{2}<0.00\right)$.

\section{Risk of Bias}

Overall, the risk of bias for the RCTs was rated low. However, for some studies it deviated from a low rating in single categories (table 3).

\section{Outcome Analyses for Observational Studies}

The 6 observational studies included in this systematic review compared various transfusion regimens: autologous pre-donation versus allogenic transfusion [25], autologous pre-donation plus peri-operative cell-saving methods versus allogenic transfusion regimen [26], allogenic transfusion versus no transfusion [27, 30], allogenic transfusion versus autologous transfusion [28] and allogenic versus autologous versus no transfusion [29].

The following outcomes were evaluated: 30-day mortality [30], TEE $[25,26]$, myocardial infarction [30], wound infection (all), pneumonia [29], and LOS [25, 26, 28, 30]. 


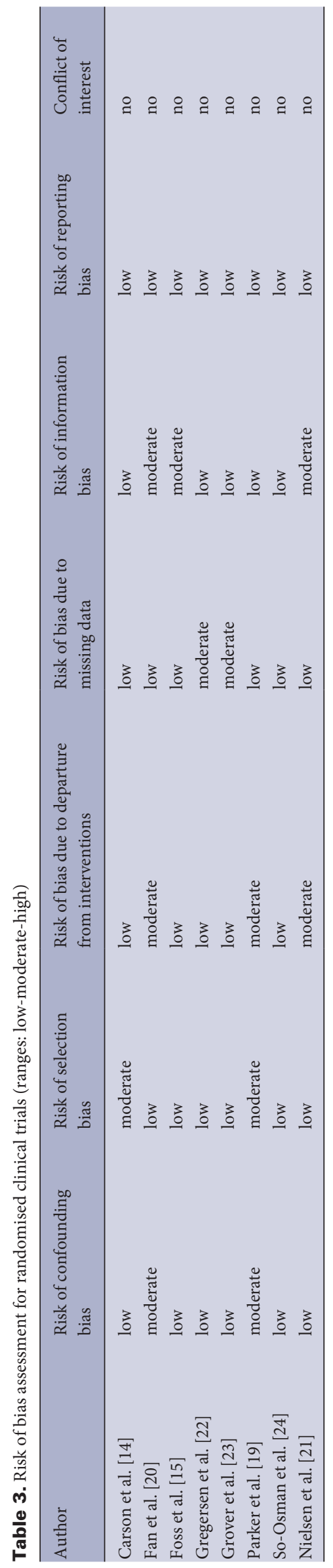

\section{Risk of 30-Day Mortality}

The only observational study which analysed 30-day mortality was performed by Klika [30]. No significant difference between transfused and non-transfused patients (not further specified in allogenic/autologous or in terms of transfusion regimen) was found.

\section{Risk of TEE}

Three studies analysed this outcome. Bierbaum et al. [25] found no association between the transfusion of blood components and incidence of TEE. In contrast, Borghi et al. [26] observed a higher incidence in patients transfused with allogenic blood transfusions compared to those who received autologous transfusions. Within the scope of a retrospective database analysis, Klika et al. [30] reported adjusted ORs of 1.77 and 1.56 for pulmonary embolism and venous thrombosis, respectively, associated with allogenic blood transfusion.

\section{Risk of Myocardial Infarction}

Only 1 study analysed this outcome. Borghi et al. [26] reported a higher incidence of myocardial infarction in the allogenic transfusion group.

\section{Risk of Wound Infection}

All observational studies analysed this outcome and all found a higher incidence for the allogenic transfusion group compared to the autologous transfusion group and no transfusion. The lowest infection rates were recorded for patients that received either autologous or no transfusion.

\section{Risk of Pneumonia}

One study, Friedman et al. [29] reported an increased incidence of pneumonia for allogenic transfusion compared to autologous or no transfusion, with the lowest risk associated to the no transfusion group.

\section{Risk of LOS}

Four out of the 6 observational studies (Bierbaum et al. [25], Borghi et al. [26], Rosencher et al. [28], Klika et al. [30]) analysed the outcome LOS. All found significant differences in favour of the no transfusion group and the non-allogenic transfusion group. Rosencher et al. [28] separately analysed the LOS for autologous transfusion versus allogenic transfusion and no transfusion and found a significant difference in favour of the autologous group.

Combined effect size calculations were not applicable for the outcomes discussed.

\section{Discussion}

In our systematic review, we analysed the short-term effects (up to 30 days) of RBC transfusion in patients with major orthopaedic surgery. We included 8 RCTs and compared associated adverse reactions following restrictive versus liberal transfusion strategies. Furthermore, 6 observational studies were reviewed. 


\section{Primary Outcomes}

In the meta-analyses for primary outcomes, no significant differences were detected between restrictive and liberal transfusion regimens. Statistical analysis did not indicate any differences for three parameters: risk of 30-day mortality, TEE and pneumonia. Regarding cardiovascular events, a (small) difference was demonstrated in advantage of the liberal transfusion regimen $(\mathrm{p}=0.08)$. In contrast, events of stroke/TIA and wound infection occurred less often under a restrictive transfusion regimen $(\mathrm{p}=0.08$ and $\mathrm{p}=0.06)$.

Of note, our findings are supported by two recently published systematic reviews with meta-analyses on blood transfusion regimens within the scope of major orthopaedic surgeries [31, 32] that did not find significant differences between the restrictive and the liberal treatment regimen regarding primary outcomes either.

Regarding myocardial infarction, in our analysis the trend in favour of the liberal regimen was predominantly influenced by the trial of Carson et al. [14]. When removing this study from the meta-analysis, the combined estimate was shifted in favour of the restrictive transfusion regimen. Within the scope of a Cochrane systematic review, Carson et al. [33] concluded 1 year later that a lower $\mathrm{Hb}$ threshold (between 7 and $8 \mathrm{~g} / \mathrm{dl}$ ) can be regarded safe nevertheless emphasising that for high-risk patients, e.g. those with acute coronary syndrome or acute renal failure, effects of a restrictive regimen had not been investigated yet. For these subgroups, evidence is still lacking from RCTs supporting a restrictive regimen, and for the time being, it may perhaps be advisable to apply a more liberal transfusion regimen. Our analysis supports the view that particularly in patients with cardiovascular risk factors a more cautious approach is warranted. Considering the meta-analysis for the outcome stroke/TIA, the observed trend in favour of a restrictive transfusion regimen was also mainly influenced by the trial of Carson et al. [14]. However, removing these data only marginally changed the combined estimate.

\section{Secondary Outcomes}

LOS did not differ between the two regimens $(\mathrm{p}=0.98)$. The average LOS varied for the restrictive regimen from study to study between 7.3 days and 23.3 days and for the liberal regimen between 7.5 and 21.8 days with the wide range reflecting strong regional differences in health care.

The only relevant difference was confirmed for the probability of receiving at least one $\mathrm{RBC}$ transfusion which was significantly increased in the liberal transfusion group. Of note, we identified a $50 \%$ reduction in the risk of receiving $\mathrm{RBCs}$ in the restrictive regimen group (in line with Mitchell et al. [32]. Under this aspect, restrictive guidelines definitely contribute to a more conscious use of RBCs without reducing the standard of patient care.

\section{Potential Biases and Limitations}

Duration of follow-up varied across the RCTs, was sometimes unclear $[15,20]$ or was not reported [23]; thus the risk of bias from missing data was rated as moderate.
In 2 studies [19, 20], relevant confounders were not measured or not appropriately analysed, thus a moderate risk for confounding bias cannot be excluded.

In 1 study [21] RBC transfusion was occasionally not performed according to protocol (in the liberal group in 3 patients; in the restrictive group in 6 patients). In another study, patients received not only RBCs but also fresh frozen plasma (FFP) [20]. In these studies, a risk for information bias cannot be excluded. In 1 study, the eligibility criteria were not clearly specified [19] and thus were open for interpretation which may have caused uncontrolled selection bias.

One study [14] was exclusively performed in patients with cardiovascular disease or with cardiovascular risk factors. To exclude selection bias in our systematic review, we performed a sensitivity analysis: By removing this study with selective inclusion of patients with pre-existing cardiac disease and/or cardiovascular risk factors [14], the combined estimates were not changed except for the outcome 'myocardial infarction' for which the combined estimate was shifted in favour of the restrictive regimen.

For the majority of RCTs, the main risks of bias were rated low. With respect to the trial of Carson et al. [14], the risk of selection bias was assessed as moderate due to the exclusive inclusion of patients with cardiovascular co-morbidity. For the trial of Fan et al. [20], the risks of confounding bias and bias due to departure from intervention were both rated as moderate, since patients in the restrictive treatment arm received more hydroxyethyl starch and Ringer lactate to achieve hemodynamic stability, whereas patients in the liberal arm more often received FFP. Moderate risks for confounding bias, selection bias and bias due to departure from intervention were detected in the trial of Parker [19].

When looking at the restrictive and liberal transfusion regimens, it should also be considered that the transfusion thresholds markedly varied between different RTCs. In 4 trials [14, 15, 20, 23], the thresholds in the restrictive and liberal regimens were at a comparable $\mathrm{Hb}$ level $<8 \mathrm{~g} / \mathrm{dl}$ and $\leq 10 \mathrm{~g} / \mathrm{dl}$, respectively. However, in 1 RCT $[17,18]$, the threshold was defined by an individual risk-based approach depending on patient's age group, individual risk factors and the time that has passed since surgery; moreover, the 'standard of care' protocols (protocol B) markedly differed between the participating centres. For the restrictive protocol (new policy protocol A), the lowest threshold for transfusion defined for the youngest patient group with no cardiovascular risk factors ( $<50$ years) was $6.4 \mathrm{~g} / \mathrm{dl}$, which was identical with the 'standard of care protocol' threshold $(\mathrm{Hb} \geq 6.4 \mathrm{~g} / \mathrm{dl})$ for ASA I patients in hospital 1 . Standard of care protocol B and restrictive protocol A were also overlapping in terms of transfusion thresholds in the peri-operative phase in two of three participating centres.

Heterogeneity was not an issue in the majority of meta-analyses (7/8), whereas a statistically significant heterogeneity was detected for the secondary outcome 'risk of receiving any RBC transfusion' $\left(\mathrm{Q}=53.64, \mathrm{df}=5, \mathrm{p}<0.01, \mathrm{I}^{2}=90.68, \tau^{2}=0.17\right)$.

Regarding the 6 observational studies, various transfusion regimens were addressed, including the use of autologous blood. The analysis of these data led to remarkable results regarding 'infection' and 'LOS'. For both outcomes, data analysis revealed advantages 
for no transfusion as compared to autologous and allogenic transfusion, with autologous transfusion being superior to allogenic transfusion. However, due to the limited control of bias, these findings should be interpreted with caution.

\section{Current Guidelines}

Restrictive transfusion thresholds as applied in most of the analysed RCTs ( $\mathrm{Hb}$ threshold $<8 \mathrm{~g} / \mathrm{dl}$ ) are comparable to those recommended by the German Medical Association in the 'Cross-Sectional Transfusion Guidelines for Therapy with Blood Components and Plasma Derivatives' (4th revised edition) [10] which recommends transfusion during acute blood loss mandatorily only for Hb levels below $6 \mathrm{~g} / \mathrm{dl}$ and between 6 and $8.0 \mathrm{~g} / \mathrm{dl}$ depending on the patient's risk profile.

Currently available data from RCTs do not support stricter guidelines. Publications aiming at safety of patients under a strict patient blood management regimen are often based on retrospective data [5] Furthermore, low-risk patients are often included for which patient blood management does not apply, resulting in a significant selection bias. Moreover, the lack of randomisation and of adjustment for risk factors is a significant source of confounding. Patient blood management tools based on restrictive transfusion policies enable recording of treatment patterns as well as detection of misuse and may also sensitise physicians for this topic. However, it should not be over-interpreted as a gold standard for risk minimisation. Under this aspect, any transfusion policy should allow for individual solutions in patients with specific risks.

\section{Conclusion}

Based on data originating from 8 RCTs, the results of this systematic review do not suggest an increased risk associated with either a restrictive or a more liberal transfusion regimen in patients undergoing major orthopaedic surgery which is in line with previous publications on this topic, including the 2015 Cochrane review on hip fracture surgery patients by Brunskill et al. [13] and the aforementioned systematic reviews published in 2017 [31, 32]. In this context, it is important to realise that our findings cannot easily be transferred to lower thresholds. Indeed, they apply only to the thresholds used in the clinical trials that were included in our meta-analyses. For patients with cardiovascular risk factors, a more liberal transfusion regimen might be advisable, whereas patients with history of stroke/TIA might benefit from a restrictive transfusion regimen. The German transfusion guidelines [10] do reflect the current scientific knowledge. No further or more restrictive recommendations are currently warranted.

\section{Disclosure Statement}

All authors confirm that there is no conflict of interest.

\section{References}

1 Hébert PC, Wells G, Blajchman MA, Marshall J, Martin C, Pagliarello G, Tweeddale M, Schweitzer I, Yetisir E: A multicenter, randomized, controlled clinical trial of transfusion requirements in critical care. Transfusion Requirements in Critical Care Investigators, Canadian Critical Care Trials Group. N Engl J Med 1999; 340:409-417.

2 Vamvakas EC: Meta-analysis of randomized controlled trials comparing the risk of postoperative infection between recipients of allogeneic and autologous blood transfusion. Vox Sang 2002;83:339-346.

3 Hopewell S, Omar O, Hyde C, Yu L-M, Doree C, Murphy MF: A systematic review of the effect of red blood cell transfusion on mortality: evidence from large-scale observational studies published between 2006 and 2010. BMJ Open 2013;3.

4 Cata JP, Wang H, Gottumukkala V, Reuben J, Sessler DI: Inflammatory response, immunosuppression, and cancer recurrence after perioperative blood transfusions. Br J Anaesth 2013;110:690-701.

5 Meybohm P, Herrmann E, Steinbicker AU, Wittmann M, Gruenewald M, Fischer D, Baumgarten G, Renner J, van Aken HK, Weber CF, Mueller MM, Geisen C, Rey J, Bon D, Hintereder G, Choorapoikayil S, Oldenburg J, Brockmann C, Geissler RG, Seifried E, Zacharowski K: Patient blood management is associated with a substantial reduction of red blood cell utilization and safe for patient's outcome: a prospective, multicenter cohort study with a noninferiority design. Ann Surg 2016;264:203-211.
6 Rohde JM, Dimcheff DE, Blumberg N, Saint S, Langa KM, Kuhn L, Hickner A, Rogers, Mary A M: Health care-associated infection after red blood cell transfusion: a systematic review and meta-analysis. JAMA 2014;311:1317-1326.

7 Khorana AA, Francis CW, Blumberg N, Culakova E, Refaai MA, Lyman GH: Blood transfusions, thrombosis, and mortality in hospitalized patients with cancer. Arch Intern Med 2008;168:2377-2381.

8 Glance LG, Dick AW, Mukamel DB, Fleming FJ, Zollo RA, Wissler R, Salloum R, Meredith UW, Osler TM: Association between intraoperative blood transfusion and mortality and morbidity in patients undergoing noncardiac surgery. Anesthesiology 2011;114:283-292.

9 Schlenke P, Spahn DR: Patient blood management: it is time to streamline targeted therapy options. Transfus Med Hemother 2015;42:72-73.

10 The Board of the German Medical Association on the Recommendation of the Scientific Advisory Board: Cross-Sectional Guidelines for Therapy with Blood Components and Plasma Derivatives. Transfus Med Hemother 2009;36:345-492.

11 Ahmed I, Chan, Julian K K, Jenkins P, Brenkel I, Walmsley P: Estimating the transfusion risk following total knee arthroplasty. Orthopedics 2012;35:71.

12 Basora M, Pereira A, Soriano A, Martínez-Pastor JC, Sánchez-Etayo G, Tió M, Salazar F: Allogeneic blood transfusion does not increase the risk of wound infection in total knee arthroplasty. Vox Sang 2010;98:124129.
13 Brunskill SJ, Millette SL, Shokoohi A, Pulford EC, Doree C, Murphy MF, Stanworth S: Red blood cell transfusion for people undergoing hip fracture surgery. Cochrane Database Syst Rev 2015;4:CD009699.

14 Carson JL, Terrin ML, Noveck H, Sanders DW, Chaitman BR, Rhoads GG, Nemo G, Dragert K, Beaupre L, Hildebrand K, Macaulay W, Lewis C, Cook DR, Dobbin G, Zakriya KJ, Apple FS, Horney RA, Magaziner J: Liberal or restrictive transfusion in high-risk patients after hip surgery. N Engl J Med 2011;365:2453-2462.

15 Foss NB, Kristensen MT, Jensen PS, Palm H, Krasheninnikoff $M$, Kehlet $H$ : The effects of liberal versus restrictive transfusion thresholds on ambulation after hip fracture surgery. Transfusion 2009;49:227-234.

16 Huedo-Medina TB, Sánchez-Meca J, Marín-Martínez F, Botella J: Assessing heterogeneity in meta-analysis: Q statistic or I2 index? Psychol Methods 2006;11:193-206.

17 So-Osman C, Nelissen R, Te Slaa R, Coene L, Brand R, Brand A: A randomized comparison of transfusion triggers in elective orthopaedic surgery using leucocyte-depleted red blood cells. Vox Sang 2010;98:56-64.

18 So-Osman C, Nelissen R, Brand R, Faber F, Slaa RT, Stiggelbout A, Brand A: The impact of a restrictive transfusion trigger on post-operative complication rate and well-being following elective orthopaedic surgery: a post-hoc analysis of a randomised study. Blood Transfus 2013;11:289-295.

19 Parker MJ: Randomised trial of blood transfusion versus a restrictive transfusion policy after hip fracture surgery. Injury 2013;44:1916-1918. 
20 Fan Y-X, Liu F-F, Jia M, Yang J-J, Shen J-C, Zhu G-M, Zhu S-H, Li W-Y, Yang J-J, Ji M-H: Comparison of restrictive and liberal transfusion strategy on postoperative delirium in aged patients following total hip replacement: a preliminary study. Arch Gerontol Geriatr 2014;59:181-185.

21 Nielsen K, Johansson PI, Dahl B, Wagner M, Frausing B, Børglum J, Jensen K, Stürup J, Hvolris J, Rasmussen LS: Perioperative transfusion threshold and ambulation after hip revision surgery - a randomized trial. BMC Anesthesiol 2014;14:89.

22 Gregersen M, Damsgaard EM, Borris LC: Blood transfusion and risk of infection in frail elderly after hip fracture surgery: the TRIFE randomized controlled trial. Eur J Orthop Surg Traumatol 2015;25:1031-1038.

23 Grover M, Talwalkar S, Casbard A, Boralessa H, Contreras M, Brett S, Goldhill DR, Soni N: Silent myocardial ischaemia and haemoglobin concentration: a randomized controlled trial of transfusion strategy in lower limb arthroplasty. Vox Sang 2006;90:105-112.

24 So-Osman C: Consequences of low postoperative haemoglobin levels on length of hospital stay after elective orthopaedic surgery in an elderly population. Vox Sang 2009;96 (suppl 1):195.
5 Bierbaum BE, Callaghan JJ, Galante JO, Rubash HE, Tooms RE, Welch RB: An analysis of blood management in patients having a total hip or knee arthroplasty. J Bone Joint Surg Am 1999;81:2-10.

26 Borghi B, Casati A: Incidence and risk factors for allogenic blood transfusion during major joint replacement using an integrated autotransfusion regimen. The Rizzoli Study Group on Orthopaedic Anaesthesia. Eur J Anaesthesiol 2000;17:411-417.

27 Garcia-Alvarez F, Al-Ghanem R, Garcia-Alvarez I, Lopez-Baisson A, Bernal M: Risk factors for postoperative infections in patients with hip fracture treated by means of Thompson arthroplasty. Arch Gerontol Geriatr 2010;50:51-55.

28 Rosencher N, Kerkkamp, Hans E M, Macheras G, Munuera LM, Menichella G, Barton DM, Cremers S, Abraham IL: Orthopedic Surgery Transfusion Hemoglobin European Overview (OSTHEO) study: blood management in elective knee and hip arthroplasty in Europe. Transfusion 2003;43:459-469.

29 Friedman R, Homering M, Holberg G, Berkowitz SD: Allogeneic blood transfusions and postoperative infections after total hip or knee arthroplasty. J Bone Joint Surg Am 2014;96:272-278.
30 Klika AK, Small TJ, Saleh A, Szubski CR, Chandran Pillai, Barsoum WK: Primary total knee arthroplasty allogenic transfusion trends, length of stay, and complications: nationwide inpatient sample 2000-2009. J Arthroplasty 2014;29:2070-2077.

31 Mao T, Gao F, Han J, Sun W, Guo W, Li Z, Wang W: Restrictive versus liberal transfusion strategies for red blood cell transfusion after hip or knee surgery: a systematic review and meta-analysis. Medicine (Baltimore) 2017;96:e7326.

32 Mitchell MD, Betesh JS, Ahn J, Hume EL, Mehta S, Umscheid CA: Transfusion thresholds for major orthopedic surgery: a systematic review and meta-analysis. J Arthroplasty 2017;32:3815-3821.

33 Carson JL, Carless PA, Hebert PC: Transfusion thresholds and other strategies for guiding allogeneic red blood cell transfusion. Cochrane Database Syst Rev 2012;4:CD002042. 